\title{
Low Voltage FESEM Evaluation of Nano and Microfabricated Materials
}

\section{E.J. Basgall}

The Pennsylvania State University Nanofabrication Facility, University Park, PA 16802

Nanofabricated materials typically have feature sizes smaller than 1um and may include; polymer resist patterns produced by electron beam (Ebeam) lithography, planarized structures from thin and thick film deposition tools, reactive ion plasma and wet etched materials, carbon and carbon-metal nanotubes. Typical materials used as substrates for nanofabrication range from conducting silicon to non-conductors or dielectrics such as glass, plastic, oxides and nitrides. This presentation addresses some of the issues to be dealt with when attempting to evaluate these novel fabricated structures using low voltage field emission scanning electron microscopy (LVFESEM). In some cases, atomic force microscopy (AFM) is used as an additional confirmatory analytical technique for verifying size measurements and surface topography.

Ebeam lithography is a multi-step process involving: 1) spin-coating a substrate with a polymer resist, 2) ebeam exposure of a computer generated pattern, 3) chemical development of the resist pattern and 4) pattern transfer by subsequent processing involving either substrate removal via an etch process or metallization and resist lift-off leaving thin metal wires on the substrate surface[1]. Electron sensitive resists used for ebeam lithography are typically spun-on between 50nm and 1000nm. These resists can either be positive or negative toned. A positive tone resist will dissolve or "clear" down to the substrate when chemically developed, forming channels. A negative tone resist will remain after development, forming raised structures.

Often, an ebeam resist is used as a mask prior to a subsequent (RIE) reactive ion etch step directed at the underlying substrate in order to transfer a pattern into the substrate material. The ratio of the etch rates between the resist and the substrate is known as the selectivity. An ideal resist/RIE recipe combination will preferentially etch the underlying substrate before degrading the resist mask [2]. Alternatively, a thin metal film is evaporated onto the patterned resist to form surface wires. The resist mask is lifted-off the substrate using solvents such as methylene chloride or acetone.

A substantial amount of work done in our laboratory involves grating fabrication for photonics applications. Ebeam lithography lends itself well to this use. Gratings have been fabricated on novel materials sometimes covered by microns of insulating oxides making conventional SEM observation difficult without applying a conducting metal coat. LVFESEM provides the ability to visualize sub micron features often without the need for a conductive metal coating. Figure 1 depicts a portion of an ebeam patterned grating in the hi resolution negative ebeam resist EN-009 (OHKA America, Inc., Somerville, NJ). The underlying substrate is silicon oxide $\left(\mathrm{SiO}_{2}\right)$ with a top coating of Si. Figure 2 depicts a cross-sectional view of a preliminary result from a planarization experiment using a PRIMAXX (Allentown, PA) deposition tool to fill in poly Si channels with $\mathrm{Ta}_{2} \mathrm{O}_{5}$. Arrows indicate voids in the deposited film where the $10 \mathrm{~nm}$ layer of $\mathrm{SiO}_{2}$ was undercut but incompletely filled.

LVSEM evaluation is a powerful tool for analyzing results of nano and microfabricated structure modification through visualization of results often unobtainable by other techniques. 


\section{References}

[1] McCord,M.A., and Rooks, M.J. Chap. 2 Electron Beam Lithography in "Handbook of Microlithography, Micromachining, and Microfabrication: Volume I"

P. Rai-Choudhury, Editor. SPIE, 1997. ISBN: 0-8194-2378-5,'1ttp://www.cnf.conell.edu/SPIEBook/toc.HTM

[2] Madou,M. Chap 2 Pattern Transfer with Dry Etching Techniques in "Fundamentals of Microfabrication" CRC Press, New York, 1997. ISBN 0-8493-9451-1



Fig. 1. LVFESEM image of a test grating in the negative ebeam resist, EN-009. Exposed at $12.5 \mathrm{uC} / \mathrm{cm}^{2}$ with a Leica EBPG-5HR lithography tool at $50 \mathrm{kV}$ accelerating voltage. Resist thickness is 200nm. Sample provided by M. Fay, Brown Univ.

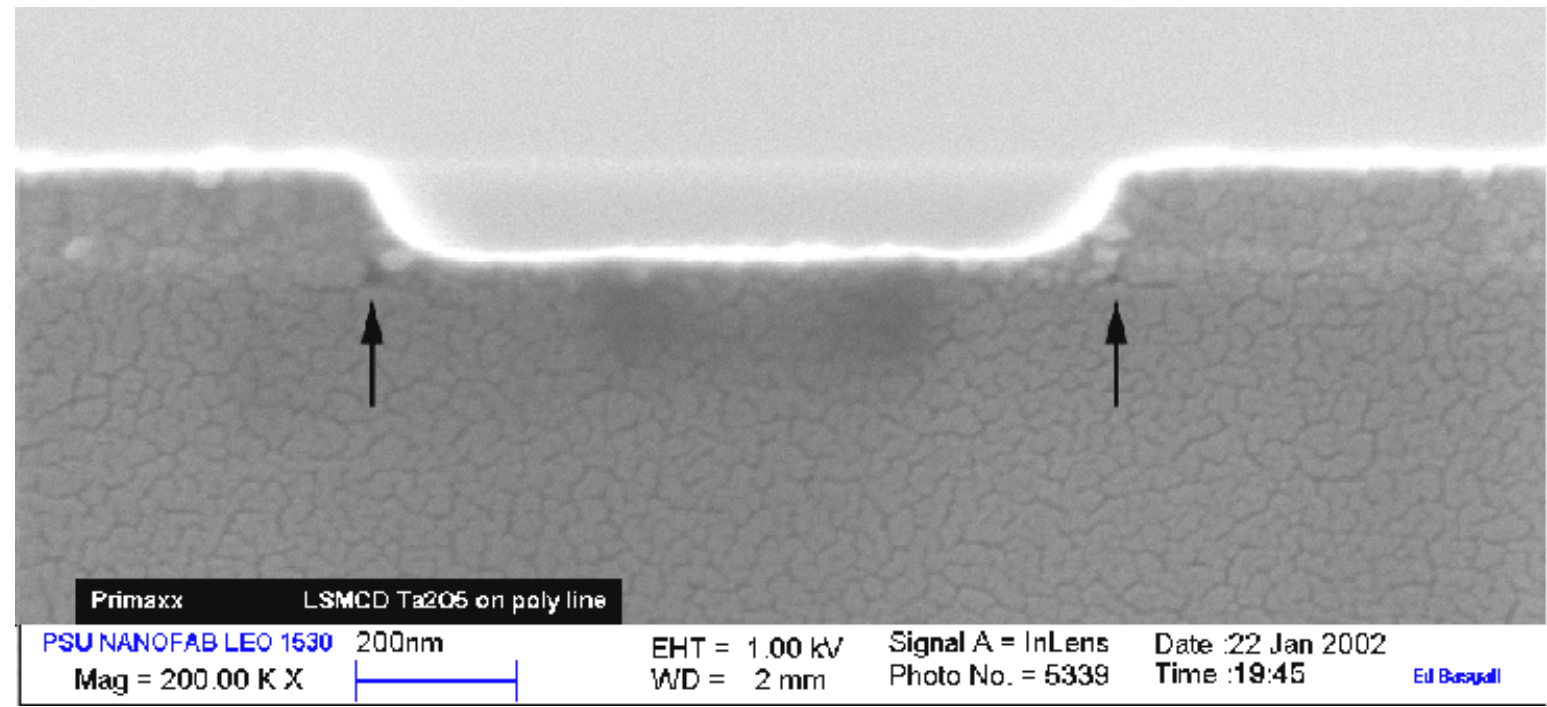

Fig. 2. LVFESEM cross-sectional image of a $\mathrm{Ta}_{2} \mathrm{O}_{5}$ film deposited onto a layered $\mathrm{Si} / \mathrm{SiO}_{2} / \mathrm{polySi} \mathrm{i}$ substrate. Sputter coated with $5 \mathrm{~nm}$ Au. Arrows indicate incomplete filling of the undercut $\mathrm{SiO}_{2}$ layer. LSMCD=liquid source misted chemical deposition. Sample provided by P. Roman, Primaxx. 